\title{
Tasty and healthy TR(i)Ps
}

\author{
The human quest for culinary pungency
}

\section{Bernd Nilius \& Giovanni Appendino}

$\mathrm{F}$ or the majority of the developed world, the hunter-gatherer lifestyle that served our predecessors for millennia is a distant memory. Yet, despite our increasingly sophisticated approach to food as top chefs compete for Michelin stars, our basic ability to taste chemical signatures and perceive flavour has not changed. In fact, in addition to sight, the ability to taste might have been one of our ancestors' most important senses, and one under strong selective pressure. From the taste and texture of a food we are able to discern much about whether it will be healthy or harmful for us, and this probably meant the difference between life and death for our predecessors. Thus, our ability to taste is a function of survival and might explain much about the otherwise-strange things that humans find palatable today.

\section{From the taste and texture of a food we are able to discern much about whether it will be healthy or harmful for us...}

This article, however, focuses not on taste per se, but on a related physiological phenomenon: chemesthesis. Chemesthetic sensations arise when chemical compounds activate receptors associated with other senses that mediate pain, touch, texture (mechanical) and thermal perception. These sensations do not fit into the traditional sense categories of taste and smell, but are often perceived as a burn-like irritation, stinging or tingling in the nose and mouth. Remarkably, we deliberately add compounds that trigger such sensations to our food, and even enjoy the pain-like sensation that they induce. The question is: why?
Various explanations, ranging from anthropology to psychology have been put forward to explain why this kind of pungency makes food more enjoyable and why humans appreciate the irritancy associated with spices. Indeed, although pungency increases the sensitivity of the oral cavity to stimulants, it actually diminishes the appreciation of taste and flavour, usurping the attention that our brain would normally give to these stimuli.

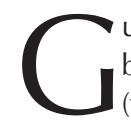
ustatory signalling starts with the binding of chemical compounds (tastants) to taste receptors, which are present on different receptor cells in the taste buds of our tongue. Taste receptors and transduction components for five basic tastes have been identified: sweet, umami (loaned from the Japanese for 'pleasant savoury taste'), bitter, salty and sour. Beyond these, taste is probably even more complex if we consider 'fatty', 'kokumi' (another Japanese word, translated as 'mouthfulness' or 'heartiness') and 'metallic' as additional qualities. In theory, by assessing food for these five qualities, we are able to consume a healthy and nutritious diet. Each type of taste corresponds to a vital constituent of food: sweetness indicates carbohydrates, 'umaminess' proteins and amino acids, and saltiness stems from important osmolytes, such as sodium chloride. A bitter taste warns us about the presence of harmful and toxic environmental compounds, while sourness tells us something about the freshness of food, allowing us to avoid rotten material.

In order to detect these tastants, humans have evolved three cell types that transmit signals to our gustatory nerve fibres (Fig 1). Taste cells are present not only on the tongue, but also throughout the mouth in solitary chemosensory cells and in the gut and the respiratory system. The taste cells on the tongue, throat and mouth synapse with afferent fibres that travel to our brain via three cranial nerves. Once they have reached the brain, taste information is further modulated by hormones and other endogenous factors. These peripheral modulations influence our preferences in terms of food intake and play important roles in regulating energy homeostasis. In addition, olfaction has a huge impact on flavour - the combination of all sensory signals about the food, which includes chemical taste signals. While the sensory systems in the palate can detect only five types of taste, it is our sense of smell, with its far greater detective range, that gives us a sensation of flavour when combined with our other senses.

\section{Various explanations, ranging from anthropology to psychology have been put forward to explain why [...] pungency makes food more enjoyable...}

However, as mentioned, our focus here is on chemesthesis, rather than taste. Thus, it is worth noting that sensory fibres from the trigeminal nerve, which express TRP (transient receptor potential) V1, TRPA1, TRPM8, TRPV4 and TRPV3 channels, innervate the whole palate. Palatine keratinocytes also express TRPV3 channels that encode heat, cold and pain sensations. They probably also encode tingling signals and might function indirectly as mechanosensitive channels. These channels, and probably more, process additional information about the chemical nature of food and modulate the activity of the real 'taste machinery', sensing 
Fig 1 | The human taste system. (A) A scheme of the three classes of taste cells. Type I cells (blue) are glia cells and act as taste-cell-supporting cells. Type 2 cells express a plethora of sweet, umami and bitter receptors (all G-protein-coupled receptors). Type 3 cells are also called presynaptic cells. They are activated by sour-activated channels, for example TRPP3, or by $\mathrm{Na}^{+}$-permeable channels, such as the epithelial $\mathrm{Na}^{+}$channel $\mathrm{ENaC}$ or TRPV1. They can also be activated by type 2 cells. Image redrawn from Chaudhari \& Roper (2010). (B) A type 2 taste cell, as shown in (A), is activated via endocannabinoids, which enhance the perception of sweetness by activation of the receptor $\mathrm{CB} 1$. By contrast, the binding of the peptide hormone leptin suppresses taste cell type 2 activity and inhibits sweet taste. (C) A cartoon of the innervation of the mouth, tongue and palate. Note that most of the chemesthetic signals exploit TRPV1, TRPA1 and TRPM8, which are expressed on probably all sensory nerve fibres of the trigeminal nerve.

the texture, temperature and fluidity of food and providing the 'mouth-feeling' during eating. Culinary pungency itself is coupled to the activation of TRPV1. Many food supplements interact with these palatine channels and provide chemesthetic information, and their flavour and use have been known for generations. The Greek historian Plutarch (ca. 45-120 BC), for example, wrote about the use of such supplements to marinate meat: "We mix oil, wine, honey, fish paste, vinegar, with Syrian and Arabian spices, as though we were really embalming a corpse for burial". Indeed there was no shortage of pungent ingredients in the cuisine of our ancestors. Ancient humans were already familiar with the piquant taste of the three main classes of dietary activators of TRP-type channels: alkamides, isothiocyanates and terpene dialdehydes.

n fact, spices have been popular throughout human history and have had a significant impact on societies and economies. In ancient Rome, for example, exotic spices were used with such profligacy that one gets the idea that actual food was, for wealthy Romans, simply a vehicle for delivering spice. Indeed, both Roman and Medieval Europe were dependent on spices, socially and economically, just as Europe is nowadays dependent on oil (Turner, 2004). Pepper, for example, which was used extensively in Roman cuisine, was imported from India in exchange for metals and silver. In fact,

A

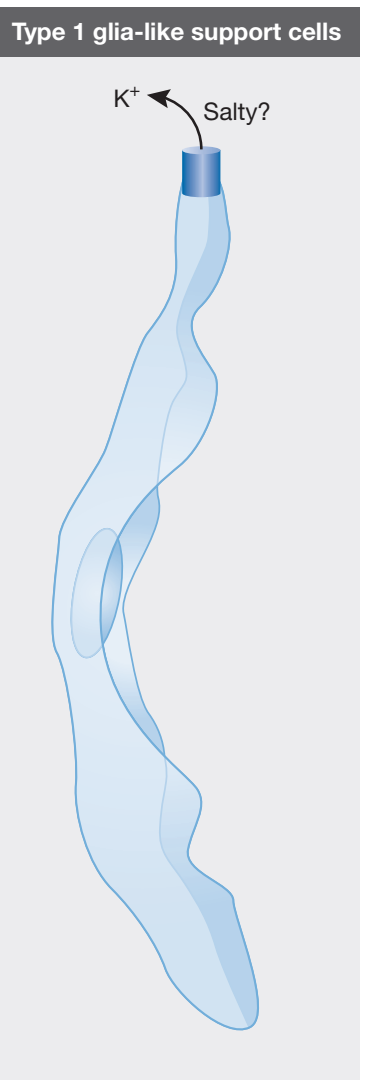

B

Type 2 receptor cells

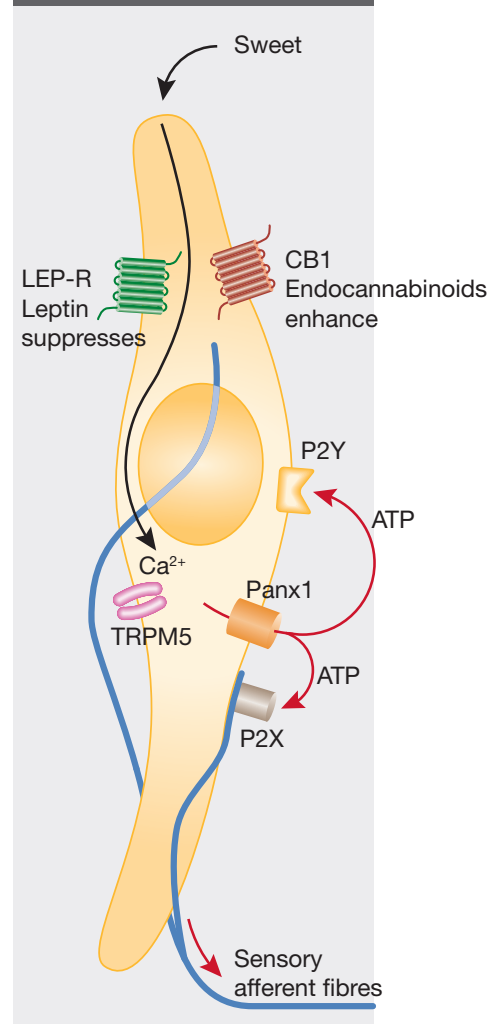

Type 3 presynaptic cells

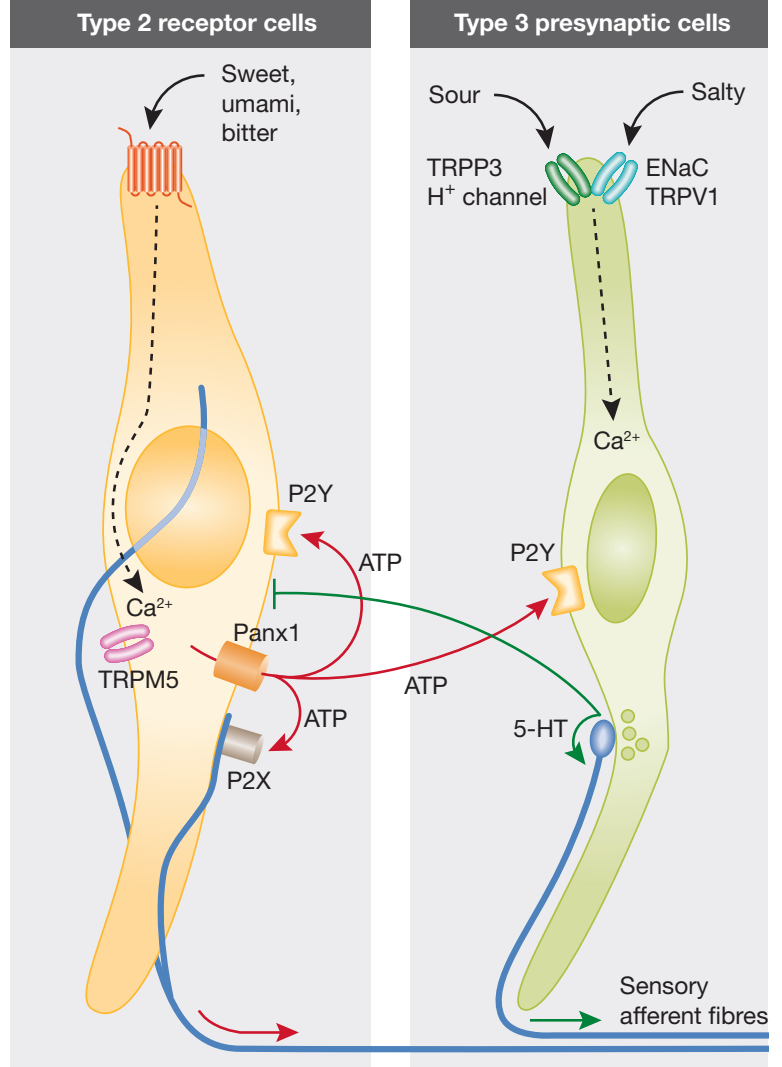

C

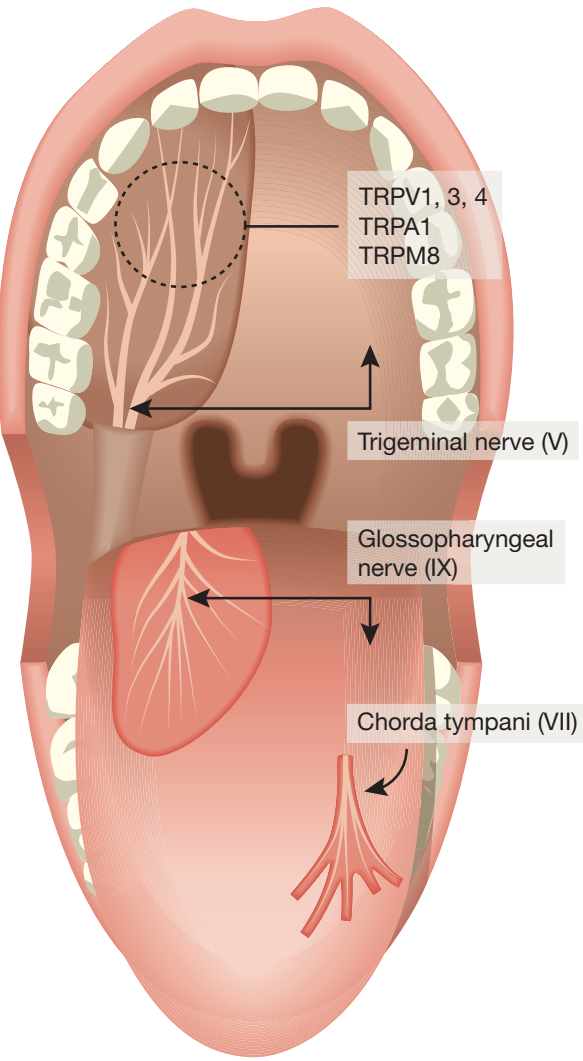


the importance of pepper cannot be overestimated. It was pepper, rather than a noble quest for discovery, that fuelled the 'Age of Exploration' and the passage from the medieval to the modern age. The goal of dominating the pepper trade was driven by the enormous amount of money that Europeans were willing to spend to activate their TRPV1 channels with piperine, the active ingredient of pepper. Thus, the discovery of America was merely a by-product of seeking a new and faster route to India. Indeed, from this perspective, one can understand why the Dutch exchanged New Amsterdam, now called New York, with the British for the tiny group of islands in the East Indies that produce nutmeg (Milton, 1999).

\section{...the reader should note that we recommend curry as preferable to licking batteries}

India and the Far East are certainly where most spices grow, so it is not surprising that eastern cuisine is so spicy. Furthermore, the cuisine of the Far East has a particular note lacking in the other cuisines of the world, namely the sensation of 'tingling'. Tingling is a curious and fascinating form of pungency, somewhat similar to the numbing sensation experienced by placing a battery on one's tongue (the reader should note that we recommend curry as preferable to licking batteries). The archetypal tingling spice is Szechuan pepper, a popular ingredient of Chinese and Japanese cuisine, and also one of the very few spices present in the cuisine of Tibet. $\alpha$-Sanshool, the active ingredient of Szechuan pepper, interacts with many sensory end points and activates TRP channels and potassium channels. At the same time, it has a similar effect on sodium channels as does the neurotoxin tetrodotoxin, resulting in overall sensory confusion. Szechuan pepper is the only spice that rivals chilli peppers for its versatility of use and has interesting applications in cosmetics, where it is popular in lipsticks - to induce a numbing sensation that mimics the expansion of the lips-and for the temporary removal of superficial wrinkles (Artaria et al, 2011).

Although modern Europeans associate spices with India and the Far East, the most celebrated and expensive spice of the GreekRoman world was silphion, which comes from the Mediterranean area. This gum-resin was obtained from a Ferula species of plant that grew around Cyrene, in today's Libya.
Weight for weight, silphion was more expensive than silver and gold. As such, it was a status symbol, associated with sexuality and purported to have both contraceptive and/or aphrodisiac properties. Sadly, silphion was driven to extinction through its consumption in the first century AD: the first documented example of the extinction of a plant by the agency of humans. Libyan silphion was eventually replaced by Persian silphion, nowadays known as asafoetida. This vile gum-resin has a faecal note that is largely lost during heating. It is especially popular in India, where it is used by Hindu sects that forbid their followers to eat vegetables that contain a sprout, such as garlic and onion. Because asafoetida is derived from a root and not a sprout, it represents a religiously correct garlic replacement (McGee, 2001).

t was the French lawyer, politician and gastronome, Jean Anthelme Brillat-Savarin (1755-1826), who was probably the first in recent history to understand the value of culinary pungency and, incidentally, to use the phrase "you are what you eat" (Fig 2). His famous 1826 book called Physiologie du Gout, ou Meditations de Gastronomie Transcendante (still available) is witty, anecdotal and enjoyable, but might shock scientists with the statement that the discovery of a new dish does more for human happiness than the discovery of a new star. Nevertheless, Brillat-Savarin does offer us an intriguing insight into the pursuit of healthy eating: the pleasure of spicy food, the value of culinary pungency and their relation to health and the enjoyment of life in general.

\section{Ancient humans were already familiar with the piquant taste of the three main classes of dietary activators of TRP-type channels}

This idea of pleasure leads us to the first possible explanation of the human quest for pungency. As omnivores, early humans had a highly diversified diet, probably dependent on the season and the availability of various berries, nuts and animals. The advent of agriculture simplified our diet to include only a few plants that did little to stimulate the human palate. In a certain sense, agriculture separated entirely the nutritional and sensory elements of the human diet such that what was good for us no longer tasted so good. In this context, spices can be viewed as concentrated sources of flavour, and our quest for them as an attempt to satisfy a sensory system primed by the diversified and flavour-rich diet of our hunter-gatherer ancestors. Alternatively, and in psychological terms, hot cuisine might be the culinary equivalent of benign masochistic activities, similar to a parachute drop, a hot bath, a cold shower, or a horror movie. All of these activities are characterized by a constrained risk-that is, a risk typical of a life-threatening event but evoked in a safe settingand the effect has been cogently named the 'rollercoaster effect'. We enjoy it because it feels harmful, but we know it is safe.

Over the past years, however, the anthro-

\section{...ancestral humans who liked spicy food-and therefore gained from its health benefits-might well have had longer, healthier lives and more offspring}

pological and psychological theories about our craving for spices have been increasingly questioned by research suggesting that evolution might have programmed us to like spices simply because they are good for our health. The classic rationale for the use of pungent spices is that they sanitize food due to their antibacterial activity-as Plutarch intimated. Spices such as pepper and cinnamon were certainly used to preserve food, especially from animal sources, but the historical decline of the use of spices in European cuisine was not accompanied by any significant improvement in food preservation, suggesting this had not been their original use. An alternative reason-and one that ties in with the use of natural remedies through the ages - is that spices were known to provide health benefits. In our modern age of molecular techniques, we now have strong preclinical evidence for the healthpromoting properties of several constituents of spices (Aggarwal, 2011a,b).

From an evolutionary point of view, food choice requires a vital decision to 'take it or leave it'. For our hunter-gatherer ancestors, this decision might well have been one of life or death. Accepting or rejecting a food is a complex process. Acceptance of food depends not only on taste, but also on olfactory, tactile and visual signals, as well as memories of previous, similar experiences and social expectations. Food palatability and hedonic value therefore play central roles in nutrient intake. As a result, 


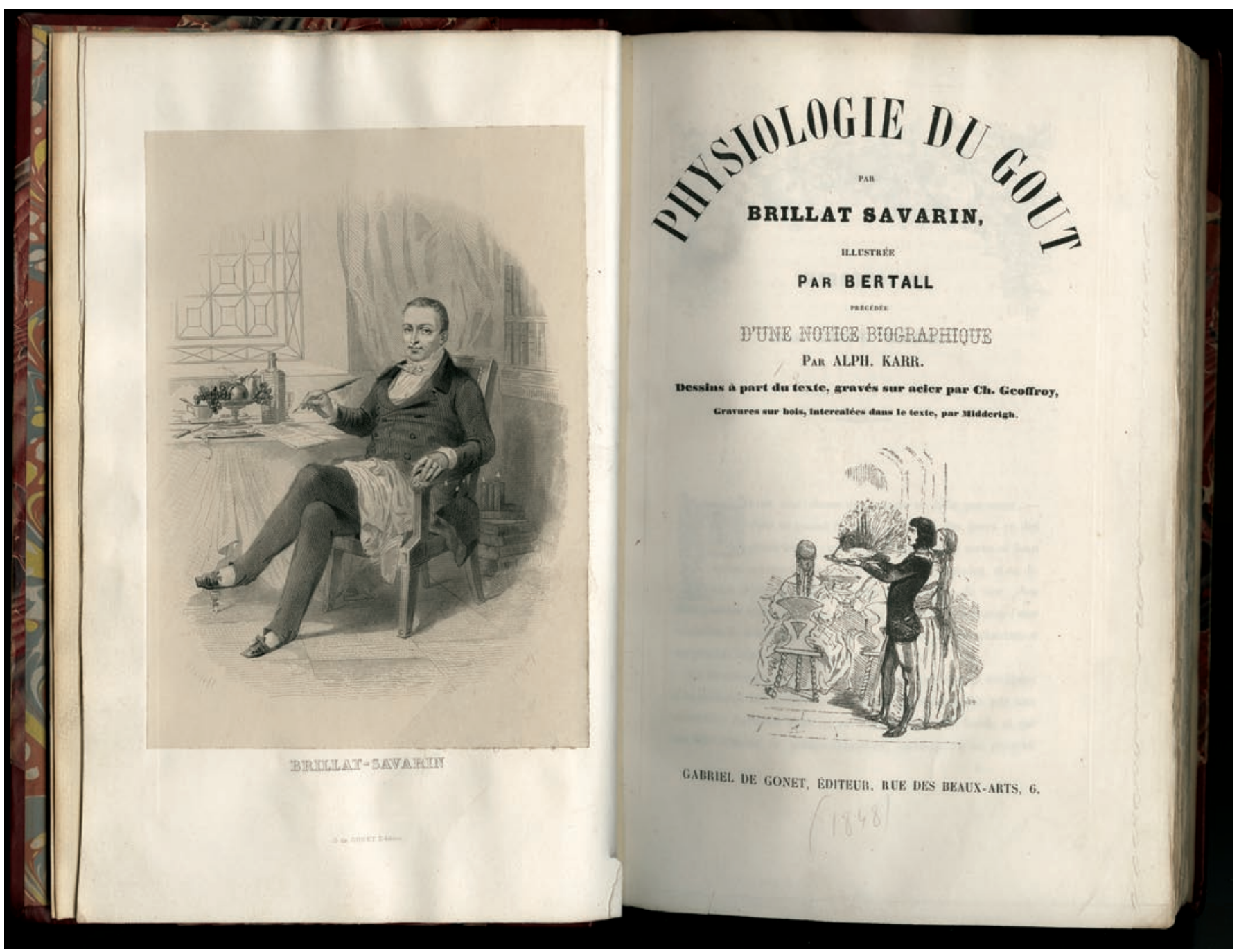

Fig 2 | The title page of Physiologie du Goût (The Physiology of Taste). This famous book was written by the French lawyer, politician and gastronome Jean Anthelme Brillat-Savarin (1755-1826). The portrait is of the author and the book includes the first formulation of phrase, "you are what you eat". This page is from the 1848 edition, printed by Gabriel De Gonet in Paris, France. Copy of an original in the Special Collections Research Center, Kelvin Smith Library, Case Western Reserve University, Cleveland, Ohio.

ancestral humans who liked spicy foodand therefore gained from its health benefits — might well have had longer, healthier lives and more offspring. Their children, in turn, would have become acquainted with the use of certain spices, spreading the habitual tolerance to offensive gustatory sensations throughout early human society.

n this modern age of molecular biology, spice compounds have proven extraordinarily complex to study because they are pleiotropic agents with both direct, receptormediated, and indirect, gene-transcriptionmediated effects. Curcumin, the principle phytochemical from turmeric (Fig 3), exemplifies these difficulties. Curcumin has more than 100 distinct molecular targets, affects the transcription of thousands of genes, and has a promiscuous pharmacophore that combines Michael-acceptor, metal-chelating and antioxidant properties. Despite evidence from more than 3,000 preclinical investigations, curcumin has never emerged from the quagmire of the health food market to become a mainstream drug. Its promiscuous binding behaviour makes it impossible to explore the chemical space around its pharmacophore, since analogues would have to be assayed against multiple molecular end points, substantially transcending the 'magic bullet' principle that permeates modern drug discovery. Furthermore, nature seems to have produced a well-honed mechanism for preventing curcumin from reaching our blood, as the compound shows dismally low oral absorption, at least in a pharmaceutical matrix (Cuomo et al, 2001).

Nevertheless, ethnobotanist James Duke, creator of an important database on phytochemicals at the US Department of Agriculture, advocates accompanying any pharmaceutical with selected spices; a suggestion already followed by thousands of cancer patients who take turmeric in addition to their mainstream treatment (Duke, 2010).

Duke argues that humans developed the ability to selectively use the phytochemicals in the plants they consumed and that, in this sense, spices are treasure troves. 


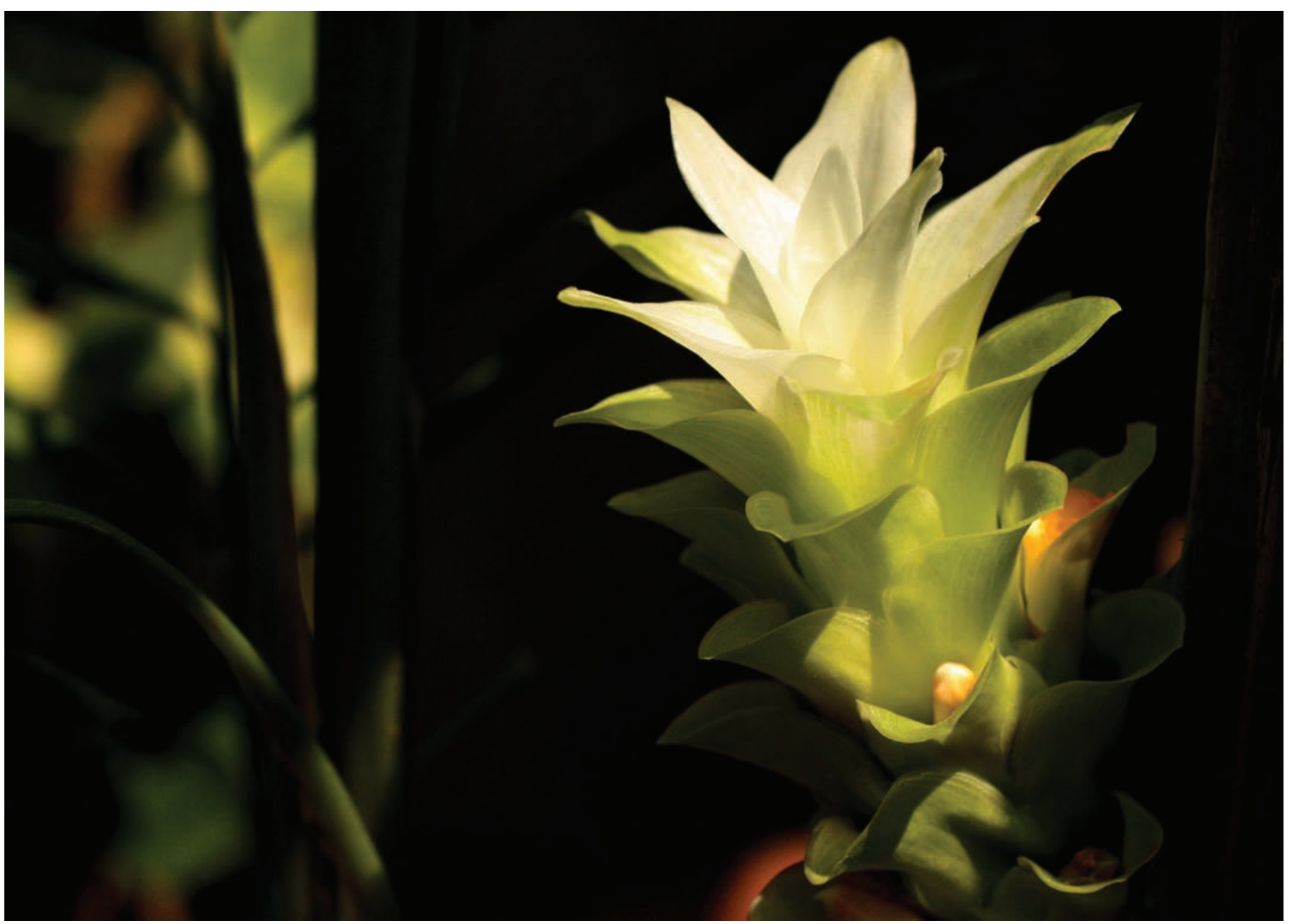

Fig 3 Turmeric flower (Curcuma longa). To the medieval Europeans, turmeric was known as Indian saffron because it was much cheaper than the real deal. The spice itself is made by boiling the plant's rhizomes for several hours and then drying and grinding them to produce the dirty yellow powder familiar to chefs worldwide. Turmeric has a slightly bitter, mildly hot and peppery flavour. It is used in eastern cuisine to add spiciness to curries and other dishes, and for the bright yellow colour it gives to food. In fact, it is used extensively throughout the world as a food colouring additive. Sadly, this is its only use. Although the active ingredient in turmeric, curcumin, is a highly biologically active molecule, it is so promiscuous that modern medicine has failed to find a way to safely take advantage of its diverse abilities. In fact, our own bodies prevent us from absorbing too much curcumin, perhaps because it has such strong effects.

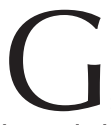
iven the lack of success with the multitalented curcumin, it is not surprising that capsaicin, a TRPV1 ligand devoid of significant affinity for any other biological target, is the only dietary TRP ligand that has reached the status of a mainstream drug. Capsaicin, along with other pungent spices such as piperazine, chilli and rutaecarpine-an alkaloid found in certain herbs including the Chinese herb Wu-Chu-Yu_seem to have extensive health benefits in relation to problems associated with being overweight.

Obesity has become a serious problem in the developed world and is associated with diabetes, hypertension, heart failure, immune deficiency and late-life dementia.
Interestingly, these spices exert hypotensive effects or protective effects against cardiac or gastrointestinal injury (Peng \& Li, 2010). The extensive ingestion of natural capsaicin agonist or chilli, for example, has been shown to decrease dyspeptic and gastroesophageal reflux disease (GERD) symptoms. The typical Asian diet, which includes rice and a range of hot spices, might therefore lower cardiometabolic risks and the prevalence of hypertension. The method of action appears to be that TRPV1 activation by dietary capsaicin enhances endotheliumdependent relaxation, an effect that is based on a TRPV1-mediated increase in nitric oxide production and that might represent a promising new therapeutic intervention for hypertension (Sessa, 2010; Yang et al, 2010). Dietary capsaicin can also reduce obesityinduced inflammation and metabolic disorders such as insulin resistance and hepatic steatosis (Kang et al, 2010): pass the chilli peppers, please.

$\mathrm{n}$ fact, it is the TRPV1 channel itself-and therefore agonists and antagonists of itthat is increasingly recognized as having a vital and multifaceted role in health. A first example is its increasingly obvious link to factors affecting obesity. Research has shown that the TRPV1 channel influences metabolism and visceral fat. Mice lacking TRPV1 exhibit only subtle alterations in body temperature, or in their responses to 
thermal challenges, but are hypometabolichave lower oxygen consumption-and hypervasoconstricted-have lower tail skin temperature. In other words, they have a distinct thermoregulatory phenotype that is coupled with a predisposition to ageassociated obesity and includes hypometabolism (Garami et al, 2011). Previous research has also shown that TRPV1 agonists prevent adipogenesis in pre-adipocytes. Thus, oral administration of capsaicin for 120 days prevented obesity in male wild-type mice, but not in TRPV1-knockout mice under a high fat diet. Spicy TRPV1 activators are therefore novel players in adipogenesis and obesity (Zhang et al, 2007; Cioffi, 2007).

A second example of the health function of TRPs is related to the avoidance of high concentrations of salt. The taste of salt triggers two divergent behavioural responses: concentrated saline solutions elicit aversion, whereas low concentrations of sodium chloride are considered enjoyable and tasty. The 'attractive' salt pathway is probably mediated via the epithelial sodium channel (ENaC), but the aversive pathway, which functions as a non-selective detector for a wide range of salts, might be coupled with TRPV1 or its variant TRPV1t (Chandrashekar et al, 2010). In this case, TRPs give us the unpleasant feeling of a badly over-salted meal. TRPV1 is also involved in detecting the bitter taste typical of high concentrations of $\mathrm{Ca}^{2+}$ and $\mathrm{Mg}^{2+}$, and sensations such as salty, metallic, astringent and sour. TRPV1 is thus involved in the avoidance of high salt concentrations, rather than in tasting salt per se.

$\mathrm{G}$ iven the potential health benefits of influencing TRPV1 activity, the search for novel TRPV1 agonists has begun. Essential oils from rose, thyme geraniol, palmarosa and tolu balsam contain constituents that activate TRPV1. These include citronellol, the main constituent of rose oil, and geraniol, the main constituent of thyme geraniol and palmarosa oils (Ohkawara et al, 2010). But what if you do not like spicy foods? How are you to activate your TRPV1 channels?

Non-pungent TRPV1 agonists represent attractive dietary ingredients to support weight loss, given the effect of capsaicin on wild-type mice consuming high-fat diets. Fortunately, the pungent properties of TRPV1 agonists and their activating potency are substantially unrelated. The pungency of TRPV1 agonists is critically dependent on lipophilicity: highly lipophilic agonists are less pungent because they cause slow TRPV1 activation, delaying or even suppressing its ability to trigger action potentials in sensory neurons (Ursu et al, 2010). Nevertheless, all other TRPV1-related effects are maintained (Chu et al, 2009), including those on thermogenesis. These considerations underlie the development of capsinoids, a group of non-pungent ester isosteres of capsaicinoids, as slimming agents. These compounds were discovered in the late 1990s in a sweet pepper cultivar (CH-19 Sweet) from Thailand (Kobata et al, 1998, 1999) and later found in high concentrations in the Japanese cultivar Himo. The production of these compounds is associated with specific mutations in the putative aminotransferase gene ( $p$-AMT) responsible for the reductive amination of vanillin, the key step in the biosynthesis of capsaicin in pepper fruits. In the absence of a functioning aminotransferase, vanillic alcohol, and not vanillamine, is produced, and this alcohol is eventually acylated to capsinoids in a striking example of biosynthetic tinkering (Tanaka et al, 2010; Lang et al, 2009). In the cultivar $\mathrm{CH}-19$ Sweet, a nonsense mutation prevents the translation of the gene (Lang et al, 2009), while in Himo, a single-nucleotide substitution results in a loss of function (Tanaka et al, 2010).

\section{Dietary capsaicin can also reduce obesity-induced inflammation and metabolic disorders such as insulin resistance and hepatic steatosis...}

The capsinoids are best represented by capsiate, the ester isostere of capsaicin. Capsiate is less pungent than capsaicin and is available as a dietary supplement in the USA and Japan for promoting weight loss in combination with diet and exercise. A study on the slimming properties of capsiate in humans found an interesting association between changes in abdominal adiposity and TRPV1 polymorphism (Snitker et al, 2009). Thus, the most pronounced slimming effects induced by capsiate were observed in individuals carrying a Val585lle mutation in TRPV1 (Val/ Val and Val/lle variants), while hardly any effect was associated with the Ile/lle variant. Remarkably, sequence variation around residue 585 of TRPV1 has been associated with insensitivity to capsaicin pungency in birds and rabbits (Jordt \& Julius, 2002). Genotypic analysis is therefore vital to identify the best candidates for capsinoid use as a polymorphism in TRPV1 could underlie negative results on thermogenesis as reported in some studies on capsiate (Galgani et al, 2010).

\section{...further research is necessary before capsinoids are touted as the modern version of the Pill of Epimenides-the magic slimming ingredient inspired by the Greek philosopher...}

Clearly, further research is necessary before capsinoids are touted as the modern version of the Pill of Epimenides - the magic slimming ingredient inspired by the Greek philosopher who was rumoured not to have eaten for 50 years and then wrote a book entitled The Happiness of Fasting (Watanabe et al, 2011).

$\mathrm{T}$ RPV1 is not the only TRP channel to have potential health effects. Pungent spices often contain ingredients that activate another member of the TRP cation family, TRPA1. Almost everyone will have experienced the pungent and irritating taste of mustard. Its active component is allyl isothiocyanate (AITC), one of the most efficient activators of TRPA1. This channel is present in the sensory nerves of the mouth cavity and the palate, where activation of these channels modulates taste. Many cultures favour the addition of garlic to many meals. The active ingredient in raw garlic is allicin, which also activates TRPA1. Allicin is electrophilic and raw garlic has been shown to protect against the development of right ventricle hypertrophy, reduce right ventricular pressure in pulmonary diseases, and act as an antihypertensive. Boiled or aged garlic, which lack allicin, have no effect.

TRPA1 is a major target of many electrophilic molecules, which have wide-ranging biological effects including influencing the expression of cytoprotective proteins involved in the elimination of harmful oxidative stress. Indeed, low doses of the principal curcuminoid of the popular Indian spice turmeric, Curcuma longa, have been shown to increase the expression of genes encoding cytoprotective proteins, including antioxidant enzymes, protein chaperones, growth factors and mitochondrial proteins (Mattson, 2008). Sensing the presence of beneficial electrophiles in our food probably involves the activation of mainly TRPA 1 channels in the 
sensory fibres of our mouth, palate and tongue. Such electrophiles are present in cruciferous vegetables such as broccoli, cauliflower, watercress, Brussels sprouts, Japanese radish, black mustard, papaya and wasabi. They all cause special taste sensations that can be exploited by good chefs.

Other plants used widely in Asian cuisine also activate TRPA1. One is Perilla frutescens, which has interesting taste and somatosensory properties. Leaves from the plant are used as foods and its seeds are used to make edible oil or to flavour soup in Korea. It is also used in traditional Chinese medicine for inducing diaphoresis, dispelling heat, strengthening stomach contractions and improving digestion. Perillaldehyde and perillaketone are among the components of the aromatic extracts from $P$. frutescens that activate TRPA1 and are responsible for the chemesthetic properties of this plant (Bassoli et al, 2009).

\section{...Roman and Medieval Europe were dependent on spices, both socially and economically, just as Europe is nowadays dependent on oil}

A new TRPA1 activator that has been used for a long time in Indonesian cusine is acetoxychavicol acetate (ACA), the main pungent component in galangal (Narukawa et al, 2010). Galangal (blue ginger) is a rhizome of plants of the genus Alpinia, with many traditional culinary and medicinal uses. It stimulates the appetite, promotes blood circulation, including in the brain, and is psychoactive. However, large quantities have been reported to induce mild visual hallucinations.

Finally, it is worth considering the beneficial roles attributed to olive oil, especially to high-quality virgin olive oil. Oleocanthal is the major anti-inflammatory phenolic in extra-virgin olive oil. It causes oral pungency sensed almost exclusively in the throat. This rare irritation pattern is a consequence of the specific activation of TRPA1 channels in sensory trigeminal fibres in the pharynx by oleocanthal. Interestingly, the 'over-the-counter', non-steroidal analgesic ibuprofen elicits the same restricted pharyngeal irritation as oleocanthal (Beauchamp et al, 2005). This example might support the decade-old idea that the taste properties of a compound predict its drug potency in the body (Peyrot des Gachons et al, 2011). The beneficial effects of lifelong consumption of olive oil, as seen in Mediterranean countries, also has an intriguing ethnological implication: Mediterraneans, who have developed a taste for the 'throaty' bite of primary olive oil, benefit from its health effects, whereas other cultures, such as the more fat and umami-oriented Americans, do not (Drahl, 2011). In this context, a study conducted by researchers at Columbia University found that people who consume the most Mediterranean food have up to a $40 \%$ lower risk of developing Alzheimer disease than those who seldom eat such food (Feart et al, 2009; Scarmeas et al, 2009).

$\mathrm{n}$ the context of healthier, longer lives, another TRP channel might also play an important role. Our ability to taste is not limited to the mouth, but also extends to the gut. The signalling cascade that allows us to taste sweet and bitter is dependent on TRPM5. This TRP channel is expressed in some gastric cells, where the activation of bitter taste receptors, and subsequently TRPM5, stimulates ghrelin secretion. Ghrelin is a hunger hormone with progastrokinetic effects. The modulation of endogenous ghrelin levels by bitter tastants - the famous German 'Magenbitter', bitter-tasting aperitifs or digestives - provide novel therapeutic applications for the treatment of weight and gastrointestinal motility disorders (Janssen et al, 2011). Indeed, bitter herbs and the liqueurs prepared from them were a mainstay of European pharmacopoeias, and some bitter compounds have now been found to activate both TRPA1 and bitter receptors, potentially providing a full range of beneficial gastrointestinal and anti-inflammatory effects.

n conclusion, spicy food not only tastes better than 'plain' food in which the five classical tastes dominate, but also often has beneficial health effects. Here we have explored how these health effects might have contributed to the development of the human taste for spicy food; a preference that social tastes and our penchant for thrilling experiences have cemented and elaborated. But the importance of spicy food does not end at the rise and fall of empires or the prevention of cancer, it also defines us as an advanced species. Unlike our animal relatives, humans have been able to move beyond eating simply for survival to eating for pleasure, for social and familial connectedness and for longer, healthier lives.
CONFLICT OF INTEREST

The authors declare that they have no conflict of interest.

\section{REFERENCES}

Aggarwal BB (2011a) Do dietary spices impair the patient-reported outcomes for stapled hemorrhoidopexy? A randomized controlled study. Surg Endosc 25: 1535-1540

Aggarwal BB (2011b) Healing Spices. New York, USA: Sterling

Artaria C, Maramaldi G, Appendino G (2011) Sichuan pepper as a "skin" spice. J Appl Cosmetol 29: 87-89

Bassoli A, Borgonovo G, Caimi S, Scaglioni L, Morini G, Moriello AS, Marzo VD, Petrocellis LD (2009) Taste-guided identification of high potency TRPA1 agonists from Perilla frutescens. Bioorg Med Chem 17: 1636-1639

Beauchamp GK, Keast RS, Morel D, Lin J, Pika J, Han Q, Lee CH, Smith AB, Breslin PA (2005) Phytochemistry: ibuprofen-like activity in extravirgin olive oil. Nature 437: 45-46

Chandrashekar J, Kuhn C, Oka Y, Yarmolinsky DA, Hummler E, Ryba NJ, Zuker CS (2010) The cells and peripheral representation of sodium taste in mice. Nature 464: 297-301

Chaudhari N, Roper SD (2010) The cell biology of taste. / Cell Biol 1906: 285-296

Chu KM, Ngan MP, Wai MK, Yeung CK, Andrews PL, Percie du Sert N, Rudd JA (2009) Olvanil: a non-pungent trpv 1 activator has anti-emetic properties in the ferret. Neuropharmacology 459: 369-375

Cioffi DL (2007) The skinny on TRPV1. Circ Res 100: 934-936

Cuomo J, Appendino G, Schneider E, McKinnon TB, Brown MJ, Togni S, Dixon BM (2001) Comparative absorption of a standardized curcuminoid mixture and its lecithin formulation. J Nat Prod 74: 664-669

Drahl C (2011) Behind olive's bite. www.cenonline.org, 21 March, pp46-47

Duke J (2010) Reductionist's rant: principles of spice therapy. I Am Herbalists Guild 9: 60-61

Feart C, Samieri C, Rondeau V, Amieva H, Portet F, Dartigues JF, Scarmeas N, Barberger-Gateau P (2009) Adherence to a Mediterranean diet, cognitive decline, and risk of dementia. JAMA 302: 638-648

Galgani JE, Ryan DH, Ravussin E (2010) Effect of capsinoids on energy metabolism in human subjects. Br Nutr / 103: 38-42

Garami A, Pakai E, Oliveira DL, Steiner AA, Wanner SP, Almeida MC, Lesnikov VA, Gavva NR, Romanovsky AA (2011) Thermoregulatory phenotype of the Trpv1 knockout mouse: thermoeffector dysbalance with hyperkinesis. / Neurosci 31: 1721-1733 Janssen S, Laermans J, Verhulst P-J, Thijs T, Tack J, Depoortere I (2011) Bitter taste receptors and a-gustducin regulate the secretion of ghrelin with functional effects on food intake and gastric emptying. Proc Natl Acad Sci USA 108: 2094-2099

Jordt SE, Julius D (2002) Molecular basis for species-specific sensitivity to "hot" chili peppers. Cell 108: 421-430

Kang JH, Tsuyoshi G, Han IS, Kawada T, Kim YM, Yu R (2010) Dietary capsaicin reduces obesityinduced insulin resistance and hepatic steatosis 
in obese mice fed a high-fat diet. Obesity (Silver Spring) 18: 780-787

Kobata K, Todo T, Yazawa S, Iwai K, Watanabe T (1998) Novel capsaicinoid-like substances, capsiate and dihydrocapsiate, from the fruits of a nonpungent cultivar, $\mathrm{CH}-19$ Sweet of pepper (Capsicum annuum L.). J Agric Food Chem 46: 1695-1697

Kobata K, Sutoh K, Todo T, Yazawa S, Iwai K, Watanabe T (1999) Nordihydrocapsiate, a new capsinoid from the fruits of a nonpungent pepper, capsicum annuum. I Nat Prod 62: 335-336

Lang Y, Kisaka H, Sugiyama R, Nomura K, Morita A, Watanabe T, Tanaka Y, Yazawa S, Miwa T (2009) Functional loss of pAMT results in biosynthesis of capsinoids, capsaicinoid analogs, in Capsicum annuum cv. CH-19 Sweet. Plant / 59: 953-961

Mattson MP (2008) Dietary factors, hormesis and health. Ageing Res Rev 7: 43-48

McGee H (2001) On Food and Cooking: the Science and Lore of the Kitchen. New York, USA: Scribner

Milton G (1999) Nathaniel's Nutmeg-How One Man's Courage Changed the Course of History. London, UK: Sceptre

Narukawa M, Koizumi K, Iwasaki Y, Kubota K, Watanabe T (2010) Galangal pungent component, 1'-acetoxychavicol acetate, activates TRPA1. Biosci Biotechnol Biochem 74: 1694-1696

Ohkawara S, Tanaka-Kagawa T, Furukawa Y, Nishimura T, Jinno H (2010) Activation of the human transient receptor potential vanilloid subtype 1 by essential oils. Biol Pharm Bull 33: 1434-1437
Peng J, Li YJ (2010) The vanilloid receptor TRPV1: role in cardiovascular and gastrointestinal protection. Eur Pharmacol / 627: 1-7

Peyrot des Gachons C, Uchida K, Bryant B, Shima A, Sperry JB, Dankulich-Nagrudny L, Tominaga M, Smith AB 3rd, Beauchamp GK, Breslin PA (2011) Unusual pungency from extra-virgin olive oil is attributable to restricted spatial expression of the receptor of oleocanthal. Neurosci J 31: 999-1009

Scarmeas N, Luchsinger JA, Schupf N, Brickman AM, Cosentino S, Tang MX, Stern Y (2009) Physical activity, diet, and risk of Alzheimer disease. JAMA 302: 627-637

Sessa WC (2010) A new way to lower blood pressure: pass the chili peppers please! Cell Metab 12: 109-110

Snitker S, Fujishima Y, Shen H, Ott S, Pi-Sunyer X, Furuhata Y, Sato H, Takahashi M (2009) Effects of novel capsinoid treatment on fatness and energy metabolism in humans: possible pharmacogenetic implications. Am / Clin Nutr 89: $45-50$

Tanaka Y, Munetaka H, Miwa T, Watanabe T, Yazawa S (2010) Newly mutated putative aminotransferase in nonpungent pepper (Capsicum annuum) results in biosynthesis of capsinoids, capsaicinoid analogues. J Agric Food Chem 58: 1761-1767

Turner J (2004) Spice: the History of a Temptation. New York, USA: Harper Collins

Ursu D, Knopp K, Beattie RE, Liu B, Sher E (2010) Pungency of TRPV1 agonists is directly correlated with kinetics of receptor activation and lipophilicity. Eur Pharmacol / 641: 114-122

Watanabe T, Ohnuki K, Kobata K (2011) Studies on the metabolism and toxicology of emerging capsinoids. Expert Opin Drug Metab Toxicol 7: 533-542

Yang D et al (2010) Activation of TRPV1 by dietary capsaicin improves endotheliumdependent vasorelaxation and prevents hypertension. Cell Metab 12: 130-141

Zhang LL et al (2007) Activation of transient receptor potential vanilloid type- 1 channel prevents adipogenesis and obesity. Circ Res 100: $1063-1070$
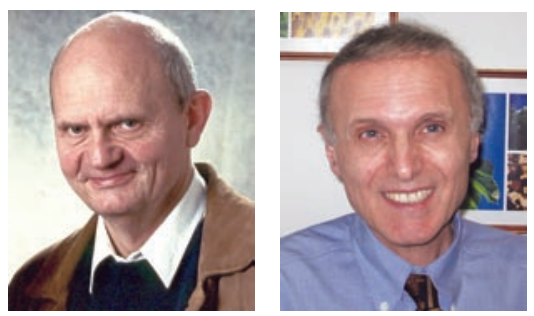

Bernd Nilius is at the KU Leuven Department of Molecular Cell Biology, Laboratory of Ion Channel Research, Belgium.

E-mail:bernd.nilius@med.kuleuven.be

Giovanni Appendino is in the Dipartimento di Scienze Chimiche Alimentari, Farmaceutiche e Farmacologiche, Novara, Italy.

E-mail:giovanni.appendino@pharm.unipmn.it

Received 20 September 2011; accepted 20 September 2011; published online 7 October 2011

EMBO reports (2011) 12, 1094-1101.

doi:10.1038/embor.2011.200 